\title{
FLIGHT CONTROL DESIGN USING BACKSTEPPING
}

\author{
Ola Härkegård * S. Torkel Glad* \\ * Division of Automatic Control, Linköping University, \\ SE-581 83 Linköping, Sweden. \\ E-mail: ola@isy.liu.se,torkel@isy.liu.se. \\ URL: http://www.control.isy.liu.se
}

\begin{abstract}
Today's prevailing nonlinear design method for aircraft flight control is feedback linearization. In this paper, backstepping is proposed as a new method to deal with the nonlinear aerodynamic forces and moments acting on the aircraft. Specifically, backstepping is used to derive state feedback control laws for angle of attack and sideslip control that require less knowledge of the lift and side forces compared to feedback linearization designs. The control laws are shown to be inverse optimal with respect to meaningful cost functionals, which guarantees that stability is preserved for a certain amount of actuator saturation.
\end{abstract}

Keywords: Aircraft control, backstepping, Lyapunov functions, inverse optimality

\section{INTRODUCTION}

The development of high performance aircraft operating at high angles of attack and at high angular rates has stimulated the interest in applying nonlinear control techniques to aircraft flight control. Currently, feedback linearization (Isidori, 1995), or nonlinear dynamic inversion (NDI), as it is often referred to in the field, is the prevailing nonlinear design method with numerous applications reported, see, e.g., (Lane and Stengel, 1988), (Enns et al., 1994), (Reiner et al., 1996).

Feedback linearization aims at cancelling the nonlinear system behavior. By using nonlinear feedback, the closed loop system is rendered linear. A drawback with this approach is that for the cancellation to be possible, all the nonlinearities involved must be known exactly. In aircraft flight control, the aerodynamic forces and moments acting on the aircraft are important sources of nonlinearity to be dealt with. In practice these can not be modeled exactly and hence, perfect cancellation is not possible.
In this paper we propose a new approach to robust aircraft flight control. Our main mathematical tool is backstepping (Krstić et al., 1995). Backstepping offers a more flexible way of dealing with nonlinearities compared to feedback linearization. Nonlinearities that act stabilizing may be kept in the closed loop system while destabilizing nonlinearities may be cancelled or dominated.

Using this freedom, we design controllers for angle of attack and sideslip control that do not require complete descriptions of the lift force and the side force respectively. The key is to rely on the generic characteristics of these forces.

To realize the control laws in terms of control surface deflections, the mapping from these deflections to the resulting aerodynamic moment needs to be inverted. Assuming a general mapping between the two, this control allocation problem is solved using nonlinear optimization. Robustness against uncertainties and model errors in the mapping is achieved by recursively estimating the bias from the nominal model and using the estimate for feedback. This can be seen as an alternative to traditional integral feedback. 


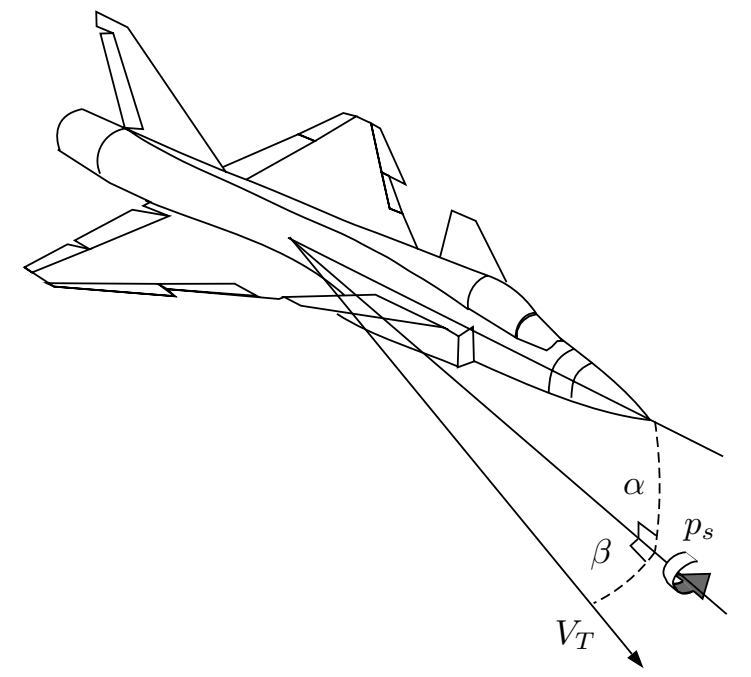

Fig. 1. Illustration of the controlled variables.

The paper is organized as follows. In Section 2 , the aircraft model to be used is presented. In Section 3, the control objectives are defined and the controller architecture is presented. The actual control design is performed in Section 4 and simulation results are shown in Section 5. Finally, Section 6 contains some concluding remarks.

\section{AIRCRAFT MODEL}

In this contribution, the controlled variables are the angle of attack, $\alpha$, the sideslip angle, $\beta$, and the roll rate about the stability $\mathrm{x}$-axis, $p_{s}$, see Fig. 1. The equations of motion describing the aircraft dynamics in terms of these variables are (Boiffier, 1998), (Stevens and Lewis, 1992):

$$
\begin{aligned}
\dot{\alpha}= & q-(p \cos \alpha+r \sin \alpha) \tan \beta \\
& +\frac{1}{m V_{T} \cos \beta}\left(-L-F_{T} \sin \alpha+m g_{1}\right) \\
\dot{\beta}= & p \sin \alpha-r \cos \alpha \\
& +\frac{1}{m V_{T}}\left(Y-F_{T} \cos \alpha \sin \beta+m g_{2}\right) \\
M= & I \dot{\omega}+\omega \times I \omega
\end{aligned}
$$

Here, $m$ is the aircraft mass, $I$ is the inertial matrix, and $V_{T}$ is the total velocity. $L$ and $Y$ are the lift and side forces respectively, $F_{T}$ is the engine thrust force, and

$$
\begin{aligned}
g_{1}= & g(\cos \alpha \cos \theta \cos \phi+\sin \alpha \sin \theta) \\
g_{2}=g & (\cos \beta \cos \theta \sin \phi+\sin \beta \cos \alpha \sin \theta \\
& -\sin \alpha \sin \beta \cos \theta \cos \phi)
\end{aligned}
$$

represent the force contributions due to gravity. These depend on the orientation of the aircraft, given by the pitch angle, $\theta$, and the roll angle, $\phi$. $M$ is the net torque applied to the aircraft and

$$
\omega=\left(\begin{array}{lll}
p & q & r
\end{array}\right)^{T}
$$

is the angular velocity of the aircraft expressed in the body axes frame.
The stability axes angular velocity

$$
\omega_{s}=\left(\begin{array}{lll}
p_{s} & q_{s} & r_{s}
\end{array}\right)^{T}
$$

is related to the body axes angular velocity through the transformation

$$
\omega_{s}=S_{\alpha} \omega, \quad S_{\alpha}=\left(\begin{array}{ccc}
\cos \alpha & 0 & \sin \alpha \\
0 & 1 & 0 \\
-\sin \alpha & 0 & \cos \alpha
\end{array}\right)
$$

The control input, $\delta$, consists of the elevator $\left(\delta_{e}\right)$, aileron $\left(\delta_{a}\right)$, and rudder $\left(\delta_{r}\right)$ deflections.

For backstepping to be applicable, we will assume these control surface deflections only to produce aerodynamic moments, and not forces. We will also neglect the derivatives of the aerodynamic forces with respect to the angular velocity ${ }^{1}$. Essentially, this yields

$$
\begin{aligned}
L(\alpha) & =\bar{q} S C_{L}(\alpha) \\
Y(\beta) & =\bar{q} S C_{Y}(\beta)
\end{aligned}
$$

where $\bar{q}=\rho V_{T}^{2} / 2$ is the aerodynamic pressure, $\rho$ is the air density, and $S$ is the wing planform area.

In the control design to come, $\dot{\omega}_{s}$, the stability axes angular acceleration, will at first be considered the control input. Introducing

$$
u=\left(\begin{array}{lll}
u_{1} & u_{2} & u_{3}
\end{array}\right)^{T}=\dot{\omega}_{s}
$$

we can rewrite the aircraft dynamics (1) as

$$
\begin{aligned}
\dot{p}_{s}= & u_{1} \\
\dot{\alpha}= & q_{s}-p_{s} \tan \beta \\
& +\frac{1}{m V_{T} \cos \beta}\left(-L(\alpha)-F_{T} \sin \alpha+m g_{1}\right) \\
\dot{q}_{s}= & u_{2} \\
\dot{\beta}= & -r_{s} \\
& +\frac{1}{m V_{T}}\left(Y(\beta)-F_{T} \cos \alpha \sin \beta+m g_{2}\right) \\
\dot{r}_{s}= & u_{3}
\end{aligned}
$$

The relationship between $u$ and the true control input, $\delta$, can be found by combining equations (1c), (2), and (3). Regarding $\alpha$ as a constant while realizing the lateral control demands $u_{1}$ and $u_{3}$ yields $u=S_{\alpha} \dot{\omega}$. Inserting this into Eq. (1c) gives us

$$
u=S_{\alpha} I^{-1}(M(\delta)-\omega \times I \omega)
$$

where we assume $M$ to be a static function of the demanded control surface deflections, $\delta$, ignoring the fast actuator dynamics.

Introducing the state vector $x=\left(\begin{array}{lllll}\alpha \beta & p_{s} & q_{s} & r_{s}\end{array}\right)^{T}$ we can use the compact form

$$
\begin{aligned}
& \dot{x}=f(x)+B u \\
& u=g(\delta, x)
\end{aligned}
$$

to describe the uncontrolled aircraft dynamics $(4)-(5)$.

\footnotetext{
1 These assumptions are the same as in feedback linearization applications.
} 


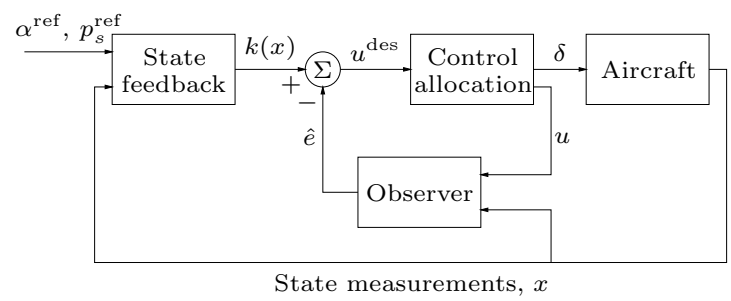

Fig. 2. Overview of the controller architecture.

\section{CONTROL PRELIMINARIES}

\subsection{Control objectives}

The angle of attack and the stability axis roll rate should follow the pilot commanded values $\alpha^{\text {ref }}$ and $p_{s}^{\text {ref }}$ respectively. A stability axis roll, also known as a velocity vector roll, is a roll performed at constant angle of attack and zero sideslip. The sideslip is to be kept zero at all times. Speed control is assumed to be handled separately.

\subsection{Controller architecture}

The block diagram in Fig. 2 gives an overview of the controller architecture that will be used.

The first block, on which this paper focuses, outputs the desired angular acceleration, $u=k(x)$. If this could be produced exactly by deflecting the control surfaces properly, the closed loop dynamics would be

$$
\dot{x}=f(x)+B k(x)
$$

In Sections 4.1 and 4.2 we derive state feedback control laws $k(x)$ such that $p_{s}=p_{s}^{\text {ref }}, \alpha=\alpha^{\text {ref }}$, and $\beta=0$ becomes a globally asymptotically stable (GAS) equilibrium of the closed loop system (7).

Realizing $u=k(x)$ requires precise knowledge of which angular acceleration, and in particular which aerodynamic moment, $M$, is produced for a certain set of control deflections, $\delta$, see (5). To allow a model error to be present in this usually quite complex mapping, we remodel the system dynamics (6) as

$$
\begin{aligned}
& \dot{x}=f(x)+B(u+e) \\
& u=\hat{g}(\delta, x)
\end{aligned}
$$

where $\hat{g}(\delta, x)$ represents our model of the mapping (5) and $e$ is the model error, which we will pragmatically model as an unknown but constant bias. Using nonlinear observer techniques (Krener and Isidori, 1983), an exponentially converging estimate, $\hat{e}$, can be produced. This estimate can be used for feedback in a straightforward way:

$$
u=k(x)-\hat{e}
$$

In (Härkegård and Glad, 2000), it is shown that closed loop stability is preserved using this adaptive control law.
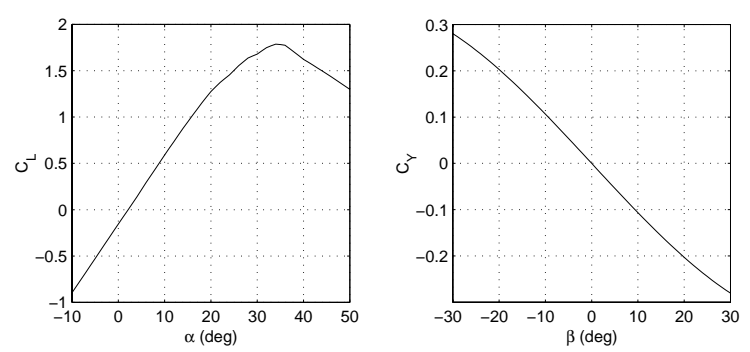

Fig. 3. Typical lift force coefficient vs. angle of attack and side force coefficient vs. sideslip.

Finally, in the control allocation block, control surface deflections are found such that $u=\hat{g}(\delta, x)$ is achieved, if possible. In the implementation, the Broyden-Fletcher-Goldfarb-Shanno (BFGS) variable metric method (Dennis and Schnabel, 1996), a quasi-Newton method, was chosen to perform the inversion numerically on-line.

\section{CONTROL DESIGN}

A general assumption that we will make is that longitudinal and lateral commands are not applied simultaneously. The mathematical effect of this is that when deriving the angle of attack control law, $\beta, p_{s}$, and $r_{s}$ are considered constant. Vice versa, when designing the sideslip and roll control laws, $\alpha$ and $q_{s}$ are considered constant. Also, due to the time-scale separation, all other variables, such as the Euler angles, $\psi, \theta$, and $\phi$, and the aircraft velocity, $V_{T}$, are considered constant.

This assumption greatly simplifies the control design by allowing us to treat the original MIMO system (4) as 3 independent SISO systems. Feedforward corrections will be used to account for the nonlinear couplings in (4), and in Section 5 simulations show that simultaneous commands are handled well, justifying the approach.

\subsection{Stability axis roll control}

Controlling the stability axis roll rate, $p_{s}$, is straightforward. Considering its dynamics in Eq. (4a), simply assign

$$
u_{1}=k_{p_{s}}\left(p_{s}^{\mathrm{ref}}-p_{s}\right)
$$

where $1 / k_{p_{s}}$ is the desired roll time constant. This corresponds to ordinary proportional control.

\subsection{Angle of attack and sideslip control}

To begin with, we note the structural similarities between the angle of attack dynamics (4b)-(4c) 


\begin{tabular}{|c|c|c|}
\hline $\begin{array}{c}\text { General system } \\
(10)\end{array}$ & $\begin{array}{c}\alpha \text { dynamics } \\
(4 \mathrm{~b})-(4 \mathrm{c})\end{array}$ & $\begin{array}{c}\beta \text { dynamics } \\
(4 \mathrm{~d})-(4 \mathrm{e})\end{array}$ \\
\hline$x_{1}^{\text {ref }}$ & $\alpha^{\text {ref }}$ & 0 \\
$x_{1}$ & $\alpha$ & $\beta$ \\
$x_{2}$ & $q_{s}$ & $-r_{s}$ \\
$u$ & $u_{2}$ & $-u_{3}$ \\
$y$ & $p_{s}, \beta, V_{T}, h, \theta, \phi$ & $\alpha, V_{T}, h, \theta, \phi$ \\
$f\left(x_{1}, y\right)$ & $f_{\alpha}\left(\alpha, y_{\alpha}\right)$ & $f_{\beta}\left(\beta, y_{\beta}\right)$ \\
\hline
\end{tabular}

Table 1. The relationships between the general nonlinear system (10) and the angle of attack and sideslip dynamics in (4b)-(4e).

and the sideslip dynamics (4d)-(4e). Both these second order systems can be written

$$
\begin{aligned}
& \dot{x}_{1}=f\left(x_{1}, y\right)+x_{2} \\
& \dot{x}_{2}=u
\end{aligned}
$$

where $y$ represents the influence of variables that we regard as constant, and $x_{1}=x_{1}^{\text {ref }}$ is the control objective. The reference, $x_{1}^{\text {ref }}$, is assumed to be constant. Table 1 summarizes the relationships between (10) and the original aircraft entities in the two cases. Here,

$$
\begin{aligned}
& f_{\alpha}\left(\alpha, y_{\alpha}\right)=-p_{s} \tan \beta \\
& \quad+\frac{1}{m V_{T} \cos \beta}\left(-L(\alpha)-F_{T} \sin \alpha+m g_{1}\right) \\
& f_{\beta}\left(\beta, y_{\beta}\right)= \\
& \quad \frac{1}{m V_{T}}\left(Y(\beta)-F_{T} \cos \alpha \sin \beta+m g_{2}\right)
\end{aligned}
$$

have been introduced.

The main characteristics of the nonlinear term $f$ in the two cases are decided by $-L(\alpha)$ and $Y(\beta)$ respectively. From Fig. 3 we see that the gradients of $-L$ and $Y$ are negative in large parts of the operating range (the only exception is the poststall behavior of the lift force). The key property of backstepping is that it allows us to benefit from these inherently stabilizing forces and not cancel them. If we do not have to cancel them, we need less information about them, which is appealing from a robustness point of view.

In a nutshell, this is where our backstepping approach differs from feedback linearization. Feedback linearization renders the closed loop system linear by cancelling $f$ using nonlinear feedback. This requires complete knowledge of $f\left(x_{1}, y\right)$ as well as $\partial f\left(x_{1}, y\right) / \partial x_{1}$. Our backstepping control law on the other hand will be linear in $x_{1}$ and $x_{2}$ and only require knowledge of $f\left(x_{1}^{\mathrm{ref}}, y\right)$ and an upper bound of the slope of $f$.

We will first derive a backstepping control law for the generic system (10) and then apply it to $\alpha$ and $\beta$ control.

4.2.1. A generic backstepping design Let us consider the system (10), assuming a general nonlinearity $f$, and determine a control law that makes $x_{1}=x_{1}^{\text {ref }}$ GAS. For convenience, we make the origin the goal state by introducing the new coordinates

$$
\begin{aligned}
\xi_{1} & =x_{1}-x_{1}^{\mathrm{ref}} \\
\xi_{2} & =x_{2}+f\left(x_{1}^{\mathrm{ref}}, y\right) \\
\varphi\left(\xi_{1}\right) & =f\left(x_{1}, y\right)-f\left(x_{1}^{\mathrm{ref}}, y\right)
\end{aligned}
$$

This yields

$$
\begin{aligned}
& \dot{\xi}_{1}=\varphi\left(\xi_{1}\right)+\xi_{2} \\
& \dot{\xi}_{2}=u
\end{aligned}
$$

Now assume that there exists a maximum slope

$$
\kappa=\max _{\substack{\xi_{1} \in \mathbb{R} \\ x_{1}^{\mathrm{ref}} \in \Omega_{r e f} \\ y \in \Omega_{y}}} \frac{\varphi\left(\xi_{1}\right)}{\xi_{1}} \leq \max _{\substack{x_{1} \in \mathbb{R} \\ y \in \Omega_{y}}} \frac{\partial f\left(x_{1}, y\right)}{\partial x_{1}}
$$

Equality holds if $x_{1}^{\text {ref }}$ is not restricted, i.e., when $\Omega_{r e f}=\mathbb{R}$. To use this property in the Lyapunov framework of backstepping, we can rewrite it as

$$
\xi_{1} \varphi\left(\xi_{1}\right) \leq \kappa \xi_{1}^{2}
$$

We now turn to the actual control design.

Step 1: In the spirit of backstepping, we start by regarding $\xi_{2}$ as the control input of Eq. (13a) and find a desired globally stabilizing "virtual" control law $\xi_{2}^{\text {des }}$, using the control Lyapunov function (clf)

$$
V_{1}=\frac{1}{2} \xi_{1}^{2}
$$

Differentiating with respect to time, we get

$$
\left.\dot{V}_{1}\right|_{\xi_{2}=\xi_{2}^{\text {des }}}=\xi_{1}\left(\varphi\left(\xi_{1}\right)+\xi_{2}^{\text {des }}\right) \leq \xi_{1}\left(\kappa \xi_{1}+\xi_{2}^{\text {des }}\right)
$$

using (15). $\dot{V}_{1}$ is made negative definite by selecting

$$
\xi_{2}^{\mathrm{des}}=-k_{1} \xi_{1}, \quad k_{1}>\kappa
$$

The resulting $\xi_{1}$ dynamics, $\varphi\left(\xi_{1}\right)-k_{1} \xi_{1}$, lie in the second and fourth quadrants only and thus, $\xi_{1}$ is stabilized.

Step 2: Continue by introducing the residual

$$
\tilde{\xi}_{2}=\xi_{2}-\xi_{2}^{\text {des }}=\xi_{2}+k_{1} \xi_{1}
$$

and rewrite the system dynamics in terms of $\xi_{1}$ and $\tilde{\xi}_{2}$.

$$
\begin{aligned}
& \dot{\xi}_{1}=\varphi\left(\xi_{1}\right)-k_{1} \xi_{1}+\tilde{\xi}_{2} \\
& \tilde{\tilde{\xi}}_{2}=u+k_{1}\left(\varphi\left(\xi_{1}\right)-k_{1} \xi_{1}+\tilde{\xi}_{2}\right)
\end{aligned}
$$

In Eq. (16b) it is not clear whether the $\xi_{1}$ components are beneficial or not. Proceeding in the usual backstepping manner, by adding a $\tilde{\xi}_{2}^{2}$ term to the clf, would lead to a control law that cancels these components. The control law would then contain $\varphi\left(\xi_{1}\right)$ and consequently require the knowledge of $f\left(x_{1}, y\right)$ for all $x_{1}$, not only at the equilibrium. As we will see, this can be avoided by also adding 
a general term $F\left(\xi_{1}\right)$, to be decided, as an extra degree of freedom. This extension of backstepping is due to (Krstić and Kokotović, 1995). This gives us

$$
V_{2}=\frac{c}{2} \xi_{1}^{2}+F\left(\xi_{1}\right)+\frac{1}{2} \tilde{\xi}_{2}^{2}, \quad c>0
$$

where $F\left(\xi_{1}\right)$ is a positive definite, radially unbounded function, satisfying

$$
F^{\prime}\left(\xi_{1}\right) \xi_{1}>0, \quad \xi_{1} \neq 0
$$

We now aim at finding a $u$ that will make $\dot{V}_{2}$ negative definite.

$$
\begin{aligned}
\dot{V}_{2}= & c \xi_{1}\left(\varphi\left(\xi_{1}\right)-k_{1} \xi_{1}+\tilde{\xi}_{2}\right) \\
& +F^{\prime}\left(\xi_{1}\right)\left(\varphi\left(\xi_{1}\right)-k_{1} \xi_{1}+\tilde{\xi}_{2}\right) \\
& +\tilde{\xi}_{2}\left(u+k_{1}\left(\varphi\left(\xi_{1}\right)-k_{1} \xi_{1}+\tilde{\xi}_{2}\right)\right)
\end{aligned}
$$

At this stage it is rewarding to make the split

$$
\varphi\left(\xi_{1}\right)=\varphi_{-}\left(\xi_{1}\right)+\kappa \xi_{1}
$$

where $\varphi_{-}\left(\xi_{1}\right)$ is guaranteed to just stay inside the second and fourth quadrants. I.e.,

$$
\xi_{1} \varphi_{-}\left(\xi_{1}\right) \leq 0
$$

We note that $\varphi\left(\xi_{1}\right)-k_{1} \xi_{1}$ is also restricted to the second and fourth quadrants. Combining this with Eq. (17) we have that

$$
F^{\prime}\left(\xi_{1}\right)\left(\varphi\left(\xi_{1}\right)-k_{1} \xi_{1}\right) \leq 0
$$

also holds. Using these relationships we get

$$
\begin{aligned}
\dot{V}_{2} \leq & -c\left(k_{1}-\kappa\right) \xi_{1}^{2}+\tilde{\xi}_{2}\left(c \xi_{1}+F^{\prime}\left(\xi_{1}\right)\right. \\
& \left.+u+k_{1}\left(\varphi_{-}\left(\xi_{1}\right)+\left(\kappa-k_{1}\right) \xi_{1}+\tilde{\xi}_{2}\right)\right)
\end{aligned}
$$

We can further simplify this expression using our design freedom. The choices

$$
\begin{aligned}
c & =k_{1}\left(k_{1}-\kappa\right) \\
F^{\prime}\left(\xi_{1}\right) & =-k_{1} \varphi_{-}\left(\xi_{1}\right), \quad k_{1}>0, F(0)=0
\end{aligned}
$$

render the final expression

$$
\dot{V}_{2} \leq-k_{1}\left(k_{1}-\kappa\right)^{2} \xi_{1}^{2}+\tilde{\xi}_{2}\left(u+k_{1} \tilde{\xi}_{2}\right)
$$

To make the right hand side negative definite, and the closed loop system GAS, we select the control law

$$
u=-k_{2} \tilde{\xi}_{2}=-k_{2}\left(\xi_{2}+k_{1} \xi_{1}\right), \quad k_{2}>k_{1}
$$

Summary: Let us summarize our results. Despite the nonlinear nature of the system (13), the linear control law (18) makes the origin a GAS equilibrium. In terms of the original state variables in (10), the control law becomes

$$
u=-k_{2}\left(x_{2}+k_{1}\left(x_{1}-x_{1}^{\mathrm{ref}}\right)+f\left(x_{1}^{\mathrm{ref}}, y\right)\right)
$$

$k_{1}$ and $k_{2}$ are design parameters restricted by

$$
k_{2}>k_{1}>\max (\kappa, 0)
$$

with $\kappa$ from (14).
Since the derived control law does not rely on exact cancellation of the system nonlinearity $f$, intuition tells us that it should possess some robustness properties. This can be confirmed using the inverse optimality tools of (Sepulchre et al., 1997). It can be shown that for the system (13), the backstepping control law (18) minimizes the cost functional

$$
\begin{aligned}
\int_{0}^{\infty}( & k_{1}\left(\varphi\left(\xi_{1}\right)-k_{1} \xi_{1}\right)^{2}+\left(\frac{k_{2}}{2}-k_{1}\right)\left(\xi_{2}+k_{1} \xi_{1}\right)^{2} \\
& \left.+\frac{1}{2 k_{2}} u^{2}\right) d t
\end{aligned}
$$

For $k_{2}>2 k_{1}$, which is a stricter condition than (20), the penalty on the state variables $\xi_{1}$ and $\xi_{2}$ becomes positive definite. Then the cost functional becomes "meaningful" and the robustness properties of nonlinear optimal control as exploited in (Glad, 1987) hold. This includes a gain margin of $\left(k_{1} / k_{2}, \infty\right)$ which guarantees that stability is preserved even when the prescribed input, $u$, cannot be produced exactly, e.g., due to actuator saturation.

4.2.2. Application to $\alpha$ and $\beta$ control Evaluating the control law (19) in the angle of attack control case using Table 1 yields

$$
u_{2}=-k_{\alpha, 2}\left(q_{s}+k_{\alpha, 1}\left(\alpha-\alpha^{\mathrm{ref}}\right)+f_{\alpha}\left(\alpha^{\mathrm{ref}}, y_{\alpha}\right)\right)
$$

For inverse optimality to hold, the parameters should be chosen according to

$$
k_{\alpha, 2}>2 k_{\alpha, 1}, \quad k_{\alpha, 1}>\max \left\{\kappa_{\alpha}, 0\right\}
$$

Since we have put no restrictions on $\alpha^{\text {ref }}$, evaluating (14) yields

$$
\kappa_{\alpha}=\max _{\substack{\alpha \in \mathbb{R} \\ y_{\alpha} \in \Omega_{y_{\alpha}}}} \frac{\partial f_{\alpha}\left(\alpha, y_{\alpha}\right)}{\partial \alpha}
$$

Similarly, in the sideslip regulation case we get

$$
\begin{gathered}
u_{3}=k_{\beta, 2}\left(-r_{s}+k_{\beta, 1} \beta+\frac{g}{V_{T}} \cos \theta \sin \phi\right) \\
k_{\beta, 2}>2 k_{\beta, 1}, \quad k_{\beta, 1}>\max \left\{\kappa_{\beta}, 0\right\}
\end{gathered}
$$

assuming that $Y(0)=0$. Evaluating (14), using the fact that the sideslip reference is always zero, yields

$$
\kappa_{\beta}=\max _{\substack{\beta \in \mathbb{R} \\ y_{\beta} \in \Omega_{y_{\beta}}}} \frac{f_{\beta}(\beta)}{\beta}
$$

$f_{\beta}$, defined in (12), is dominated by the side force, $Y(\beta)$, which resides in the second and fourth quadrants, see Fig. 3. Thus $\kappa_{\beta}<0$ and the parameter restriction $k_{\beta, 1}>0$ becomes active. Note that the $u_{3}$ dependence on the side force is hereby completely removed.

\section{SIMULATIONS}

We evaluate the control laws using Admire, a Matlab/Simulink environment for the Generic 

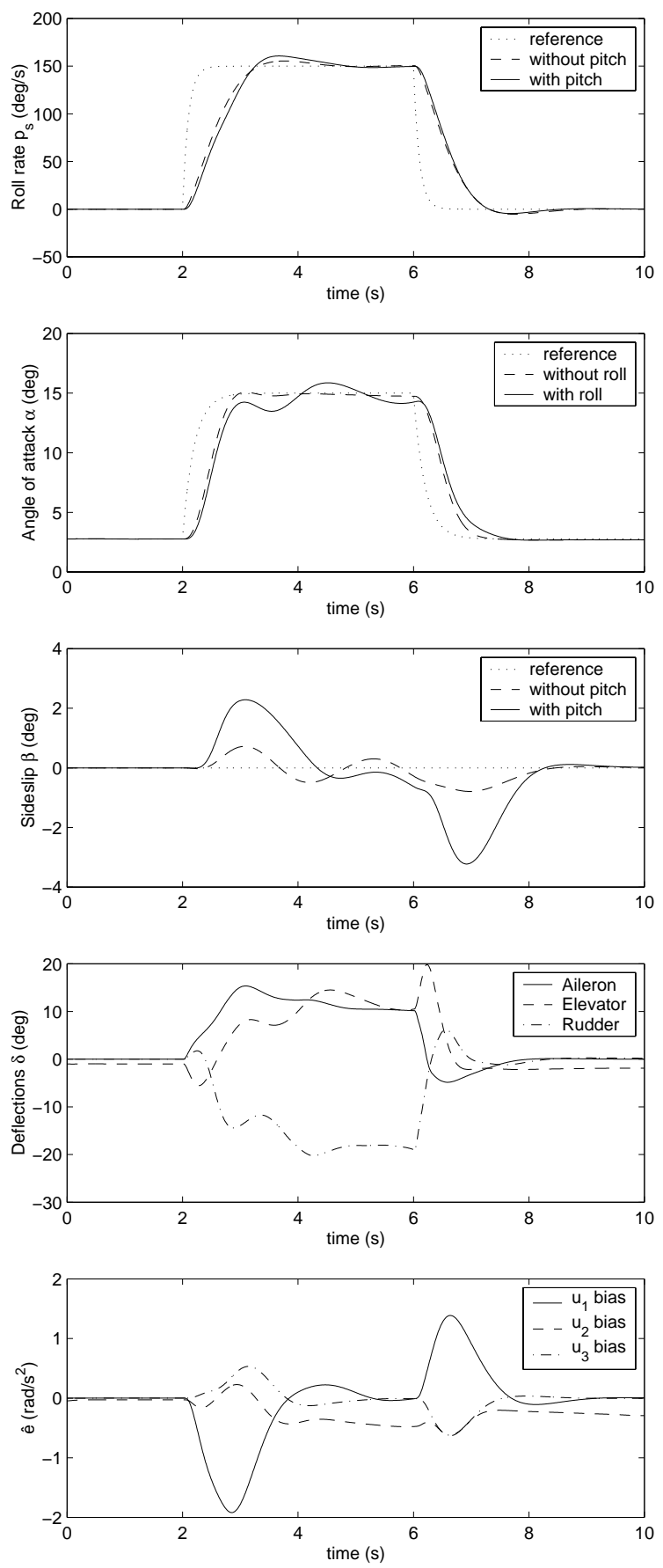

Fig. 4. Simulated aircraft responses to pitch and stability axis roll commands.

Aerodata Model (GAM) (Backström, 1997), a small generic fighter aircraft, developed by Saab AB, Sweden. The simulations are performed at an initial speed of 0.5 Mach at an altitude of $1000 \mathrm{~m}$. The control law parameters were set according to $k_{p_{s}}=k_{\alpha, 1}=k_{\beta, 1}=2, k_{\alpha, 2}=k_{\beta, 2}=5$. The poles of the bias observers were placed in $-8 \pm i$. Fig. 4 shows the responses to a sole angle of attack command, a sole stability axis roll command, and the combination of the two. The two lower graphs show the control surface delfections for the combined maneuver along with the bias estimates used in Eq. (8).

\section{CONCLUSIONS}

In this paper we have demonstrated the potential of using backstepping techniques to design flight control laws. In comparison to feedback linearization, the control laws can be made computationally simpler by not cancelling the beneficial nonlinear parts of the lift and side forces. We have also shown the control laws to solve meaningful optimal control problems which ensures a certain amount of robustness.

\section{REFERENCES}

Backström, H. (1997). Report on the usage of the Generic Aerodata Model. Technical report. Saab Aircraft AB.

Boiffier, J.-L. (1998). The Dynamics of Flight: The Equations. John Wiley \& Sons.

Dennis, Jr., J. E. and R. B. Schnabel (1996). Numerical Methods for Unconstrained Optimization and Nonlinear Equations. SIAM.

Enns, D., D. Bugajski, R. Hendrick and G. Stein (1994). Dynamic inversion: an evolving methodology for flight control design. International Journal of Control 59(1), 71-91.

Glad, S. T. (1987). Robustness of nonlinear state feedback - a survey. Automatica 23(4), 425435.

Härkegård, O. and S. T. Glad (2000). Control of systems with input nonlinearities and uncertainties: an adaptive approach. Technical Report LiTH-ISY-R-2302. Department of Electrical Engineering, Linköpings universitet. SE-581 83 Linköping, Sweden.

Isidori, A. (1995). Nonlinear Control Systems. third ed.. Springer.

Krener, A. J. and A. Isidori (1983). Linearization by output injection and nonlinear observers. Systems \& Control Letters 3, 47-52.

Krstić, M. and P. V. Kokotović (1995). Lean backstepping design for a jet engine compressor model. In: Proceedings of the 4th IEEE Conference on Control Applications. pp. 10471052.

Krstić, M., I. Kanellakopoulos and P. Kokotović (1995). Nonlinear and Adaptive Control Design. John Wiley \& Sons.

Lane, S. H. and R. F. Stengel (1988). Flight control design using non-linear inverse dynamics. Automatica 24(4), 471-483.

Reiner, J., G. J. Balas and W. L. Garrard (1996). Flight control design using robust dynamic inversion and time-scale separation. Automatica 32(11), 1493-1504.

Sepulchre, R., M. Janković and P. V. Kokotović (1997). Constructive Nonlinear Control. Springer.

Stevens, B. L. and F. L. Lewis (1992). Aircraft Control and Simulation. John Wiley \& Sons. 